\title{
Safety and costs analysis of early hospital discharge after brain tumour surgery: a pilot study
}

Iuri Santana Neville ${ }^{1,2^{*}}$, Francisco Matos Ureña ${ }^{2}$, Danilo Gomes Quadros ${ }^{2}$, Davi J. F. Solla ${ }^{2}$, Mariana Fontes Lima ${ }^{3}$, Claudia Marquez Simões ${ }^{1}$, Eduardo Vicentin ${ }^{4}$, Ulysses Ribeiro Jr ${ }^{1,5}$, Robson Luis Oliveira Amorim²,6, Wellingson Silva Paiva ${ }^{2}$ and Manoel Jacobsen Teixeira ${ }^{2}$

\begin{abstract}
Background: A daily algorithm for hospital discharge (DAHD) is a key point in the concept of Enhanced Recovery After Surgery (ERAS) protocol. We aimed to evaluate the length of stay (LOS), rate of complications, and hospital costs variances after the introduction of the DAHD compared to the traditional postoperative management of brain tumour patients.

Methods: This is a cohort study with partial retrospective data collection. All consecutive patients who underwent brain tumour resection in 2017 were analysed. Demographics and procedure-related variables, as well as clinical outcomes, LOS and healthcare costs within 30 days after surgery were compared in patients before/pre-implementation and after/postimplementation the DAHD, which included: stable neurological examination; oral feeding without aspiration risk; pain control with oral medications; no intravenous medications. The algorithm was applied every morning and discharge was considered from day 1 after surgery if criteria was fulfilled. The primary outcome (LOS after surgery) analysis was adjusted for the preoperative performance status on a multivariable logistic regression model.

Results: A total of 61 patients were studied (pre-implementation 32, post-implementation 29). The baseline demographic characteristics were similar between the groups. After the DAHD implementation, LOS decreased significantly (median 5 versus 3 days; $p=0.001$ ) and the proportion of patients who were discharged on day 1 or 2 after surgery increased (44.8\% vs 3.1\%; $p$ <.001). Major and minor complications rates, readmission rate, and unplanned return to hospital in 30-day followup were comparable between the groups. There was a significant reduction in the median costs of hospitalization in DAHD group (US\$2135 vs US\$2765, $p=0.043$ ), mainly due to a reduction in median ward costs (US\$922 vs US\$1623, $p=0.009$ ).

(Continued on next page)
\end{abstract}

\footnotetext{
* Correspondence: iurineville@yahoo.com.br

Presentation at a conference:Title: Safety and costs analysis of a "Fast-track" algorithm for early hospital discharge after brain tumour surgeryOral

Presentation Date and Time: 10/22/2019, 4:40 PM - 4:46 PM 2019 Congress of Neurological Surgeons Annual Meeting, October 19-23, in San Francisco, CA.

${ }^{1}$ Instituto do Cancer do Estado de São Paulo do Hospital das Clinicas da Faculdade de Medicina da Universidade de São Paulo, Av Dr Arnaldo 251 Cerqueira Cesar, CEP, São Paulo 01246-000, Brazil

${ }^{2}$ Division of Neurosurgery, Hospital das Clinicas da Faculdade de Medicina da Universidade de São Paulo, Av Dr Arnaldo 251 Cerqueira Cesar, CEP, São Paulo 01246-000, Brazil

Full list of author information is available at the end of the article
}

(c) The Author(s). 2020 Open Access This article is licensed under a Creative Commons Attribution 4.0 International License, which permits use, sharing, adaptation, distribution and reproduction in any medium or format, as long as you give appropriate credit to the original author(s) and the source, provide a link to the Creative Commons licence, and indicate if changes were made. The images or other third party material in this article are included in the article's Creative Commons licence, unless indicated otherwise in a credit line to the material. If material is not included in the article's Creative Commons licence and your intended use is not permitted by statutory regulation or exceeds the permitted use, you will need to obtain permission directly from the copyright holder. To view a copy of this licence, visit http://creativecommons.org/licenses/by/4.0/ The Creative Commons Public Domain Dedication waiver (http://creativecommons.org/publicdomain/zero/1.0/) applies to the data made available in this article, unless otherwise stated in a credit line to the data. 
(Continued from previous page)

Conclusions: Early discharge after brain tumour surgery appears to be safe and inexpensive. The LOS and hospitalization costs were reduced without increasing readmission rate or postoperative complications.

Keywords: Brain tumours, Discharge, ERAS, Enhanced recovery after surgery, Postoperative length of stay

\section{Background}

Until early 1990s, perioperative care was based on empirical concepts and common practice, in part due to the paucity of scientific evidence. With the need of improving patient outcomes and reducing costs, the concern of developing safe and effective standards in postoperative care emerged, and advances have been achieved. In 1994, Engelmann and colleagues introduced the concept of "Fast-Track Surgery" to optimize postoperative recovery, reduce inpatient stay and related complications [1, 2]. In 2001, the creation of ERAS (Enhanced Recovery After Surgery) group represented a paradigm shift in protocols of care to patients undergoing gastrointestinal surgery [3]. In the following years, numerous guidelines were developed by other surgical fields (bariatric surgery, hepatectomy, head and neck cancer surgery, breast reconstruction surgery, among others), which are gradually transitioning to an outpatient-based paradigm [2, 4].

Even though many of these concepts are well stablished in literature, they are not widespread among neurosurgeons in their clinical practice. Traditionally, many neurosurgical centres still adopt an in-patient postoperative care with a median of 4 days after craniotomy (such as brain tumour resection and aneurysm clipping) for safety reasons, even in cases with no perioperative complications [5].

From 2008 to 2016, outpatient brain and spinal surgery experiences have been reported [6-8]. Despite the encouraging results, only after 2015 a significant number of publications on the subject containing neurosurgical patients have arisen, including one randomized clinical trial evaluating the implementation of the ERAS protocol [4, 9-11].

Recently, our institution, aiming to standardize hospital discharge, has adopted a daily algorithm for hospital discharge (DAHD), which is a key point in the concept of ERAS protocol. Thus, we designed a retrospective cohort study to evaluate whether there was a difference in length of stay (LOS), rate of complications, and hospital costs after the introduction of the DAHD compared to the traditional postoperative management of patients who underwent brain tumour resection.

\section{Methods}

\section{Study design and patient population}

This is a cohort study with partly retrospective data collection, conducted on a sequential period at the Instituto do
Cancer do Estado de São Paulo (ICESP), University of Sao Paulo, Sao Paulo - Brazil. We have included all consecutive patients who underwent brain tumour resection during 2017 by a single neurosurgeon. Patients undergoing surgery for hydrocephalus, hematoma, cerebrospinal fluid leakage, surgical site infection, cranial reconstruction, endoscopic approach, and stereotactic biopsy were excluded so that we were focusing mainly on the subgroup of patients who underwent brain tumour resection by craniotomy. This retrospective patient medical record data review was approved by the local Ethics and Research Committee (CAAE 87064218.2.0000.0065).

\section{Daily algorithm for hospital discharge (DAHD)}

The DAHD was recently introduced as an alternative to traditional management and classical discharge criteria of our institution. The DAHD (Fig. 1) is similar to the one proposed by Sughrue et al., 2015, [4] that includes: 1) neurological status before and after surgery; 2) Oral feeding without aspiration risk; 3) Pain control with oral medications; 4) Intravenous medications not needed; and 5) Stable neurological examination. The rationale of this algorithm was to consider hospital discharge from the day 1 after surgery if patient fulfilled the criteria. The algorithm was applied every morning during our routine round. If the patient did not meet a single criterion, he was kept inpatient and re-evaluated on the next day. Therefore, the patient was only kept inpatient if he objectively presented a real need for hospital care. Postoperative blood tests and head computed tomography were performed within $24 \mathrm{~h}$ and repeated as needed.

Two study groups were selected: 1) Pre-DAHD, retrospective, comprising patients who underwent brain tumour resection between January and August 2017, and 2) DAHD, which prospectively included patients operated between September and December 2017, after the implementation of the protocol.

\section{Anaesthetic and perioperative management}

The perioperative management has not changed during the entire study period. All cases were discussed at the multidisciplinary tumour board meeting of our institution. Patients perform preoperative workup that includes basic laboratory tests, chest X-Rays, EKG, and preoperative evaluation by a general practitioner and an anaesthesiologist. 


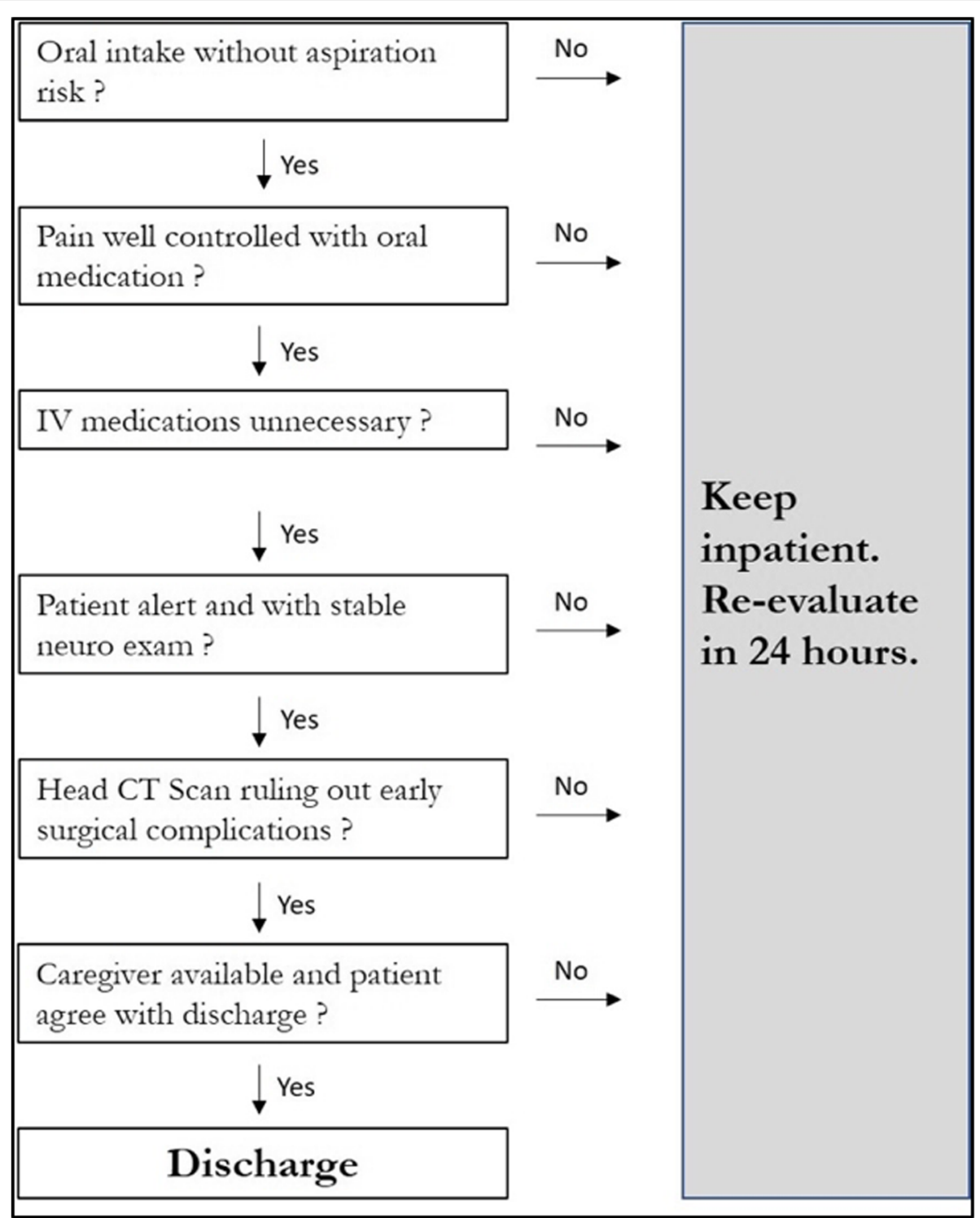

Fig. 1 Daily Algorithm for Hospital Discharge (DAHD) - This algorithm includes all the criteria that each patient had to fulfill to be discharged. If any of these were not met, the participant was kept inpatient and re-evaluated the next day

Preoperative fasting time of $8 \mathrm{~h}$, and anti-thrombotic prophylaxis with graduated compression stockings and intermittent pneumatic compression, were always applied.

The type of anaesthesia, amount of fluid, and intraoperative management of neuromuscular blockade were left to the discretion of the attending anaesthesiologist and conventional total intravenous anaesthesia was used for general anaesthesia in patients who did not underwent awake craniotomy. Patients were administered a single dose of prophylactic antibiotics (second generation cephalosporin) $30 \mathrm{~min}$ before skin incision and scalp infiltration with $10 \mathrm{ml}$ of $2 \%$ lidocaine was commonly used.
All patients underwent surgery by the smallest possible craniotomy that allowed a safe brain tumour removal. Perioperative brain mapping with navigated transcranial magnetic stimulation, neuronavigation, and intraoperative ultrasound were used when necessary.

The patients were preferably extubated in the operating room whenever possible and directly transferred to the postoperative intensive care unit (ICU). Patients were not extubated in the OR only if there were brain oedema leading to transcalvarian herniation after tumour removal, unexpected need to expand the craniotomy, or massive blood transfusion. Duration of surgery was not criteria to not extubate the patient. Postoperative dexamethasone and phenytoin doses were 
maintained as the preoperative schedule and weaning was managed case by case.

Oral feeding was reintroduced 6 hours after surgery for patients without dysphagia and with stable neurologic exam. Arterial lines, central venous catheters, and Foley bladder catheter were usually removed early the following morning (first postoperative day) and discharge from ICU was left to the discretion of the critical care staff physicians.

\section{Treatment costs}

From each patient, all the costs comprising the surgery until the 30th postoperative day were collected. For this costs assessment, the absorption costing method was applied and included the costs of infrastructure and human and material resources used. For estimation of costs, the micro-costing method, which involves the direct measurement of every input consumed in the treatment of a particular patient, was adopted. The costs are shown in US dollars, converted from Brazilian reals (1US\$ = R\$3.846; Source: Central Bank of Brazil, October 24th 2018)

\section{Data collection and statistical analysis}

Patient demographic data, tumour characterization, general performance and neurological status, histologic diagnosis, complications, and clinical outcomes until 30 days after surgery were retrieved from patients' medical records.

Categorical variables are presented as absolute frequencies and proportions and compared by means of the Pearson chi-squared or Fisher's tests. The distribution of the continuous variables was verified by graphical and statistical (skewness and kurtosis) methods. These are presented as means and standard deviations or medians and quartiles and compared through the Student $\mathrm{T}$ test or Mann-Whitney test, as appropriate.

The primary outcome (length of stay) analysis was adjusted for the preoperative Karnofsky performance status (KPS) on a multivariable logistic regression model. We did not include post-operative complications on the multivariable adjustment.

All tests were two-tailed and final $p$-values $<0.05$ were considered statistically significant. All analyses were performed on the software Statistical Package for Social Sciences, version 24.0 (SPSS, IBM Statistics, Armonk, NY, USA).

\section{Results}

A total 66 patients were included, 32 on the Pre-DAHD and 34 on the DAHD group as depicted in Table 1. There were no differences between the two groups with respect to age, gender, BMI, ASA physical status, Karnofsky Performance Scale (KPS), and Eastern
Cooperative Oncology group (ECOG) scores. Comorbidities such as hypertension, diabetes, and active smoking status were equally distributed between the two groups. In relation to preoperative clinical status, the majority of patients had no motor deficits, alteration of the level of consciousness, nor dysphagia.

In respect to tumour characteristics and surgery performed, no significant differences between Pre-DAHD and DAHD groups were found (Table 2). High-grade glioma was the most common histology in Pre-DAHD group $(n=14,43.8 \%)$, while metastasis comprised the majority of the patients of the DAHD group $(n=16$, $47.1 \%)$, although not statistically significant $(p=0.455)$. The two groups were similar with relation to previous adjuvant treatment, number of lesions resected, deep seated lesions, infratentorial location, laterality, primary motor cortex involvement, presence of oedema, and cerebral herniation. The median of the larger lesion diameter was 47.5 (quartiles 32.3-58.0) and 41.0 (quartiles 30.0-52.5) millimetres in Pre-DAHD and DAHD groups, respectively $(p=0.345)$.

There was no significant difference between the two groups in terms of surgery characteristics: the median duration of surgery was 213 (quartiles 155-298) vs 218 (quartiles 169-283) minutes in Pre-DAHD and DAHD groups, respectively $(p=0.773)$. Emergency surgeries and awake craniotomies comprised the minority of the cases and were equally distributed in both groups.

Relevant measured outcomes are shown in Table 3. In the majority of cases, gross total resection was achieved (78.8\%) with no new motor deficits $(67,7 \%)$ and these findings were similar between the two groups $(p=0.352$ and $p=0.928$, respectively). Following surgery, a higher percentage of patients in DAHD group were discharged within $48 \mathrm{~h}(47.1 \%$ vs $3.1 \%, p<0.001)$. Conversely, the postoperative median length of stay (LOS) was lower in DAHD group at 3 (quartiles 2-5) days vs 5 (quartiles 48 ) days in Pre-DAHD group $(p=0.001)$ (Fig. 2). The median ICU stay was similar between the groups: 28.8 (quartiles 22.7-67.1) hours in Pre-DAHD and 26.5 (quartiles 23.5-50.9) hours in DAHD group ( $p=0.663$ ), although there was an increase in the proportion of LOS after surgery spent in ICU in DAHD group ( 0.44 [quartiles $0.33-0.65$ ] vs 0.25 [quartiles $0.15-0.46$ ], $p=0.030$ ).

The shorter LOS observed in DAHD group was maintained after multivariable adjustment for Pre-DAHD vs DAHD group, $p=0.002$ (Table 4).

Complications and safety outcomes are displayed in Table 5. Reoperations, major and minor complications, return do the emergency department, readmissions, and deaths until the 30th postoperative day were similar between the groups. All but two patients with minor or major complications were discharged beyond the 2nd POD, both cases with minor complications (one urinary 
Table 1 Characterization of the patient and preoperative clinical status

\begin{tabular}{|c|c|c|c|c|}
\hline Variables & General (66) & Pre-DAHD (32) & DAHD (34) & $p$ \\
\hline Age (mean \pm standard deviation) & $51.5 \pm 13,8$ & $53.9 \pm 12.5$ & $49.2 \pm 14.7$ & $0.165^{a}$ \\
\hline Female Gender & $39(59.1)$ & $14(43.8)$ & $13(38.2)$ & 0.649 \\
\hline Ethnics & & & & 0.854 \\
\hline Caucasian & $32(53.3)$ & $15(50.0)$ & $17(56.7)$ & \\
\hline Brown (mulatto) & $21(35.0)$ & $11(36.7)$ & $10(33.3)$ & \\
\hline African-American & $7(11.7)$ & $4(13.3)$ & $3(10.0)$ & \\
\hline \multicolumn{5}{|l|}{ Comorbidities } \\
\hline Body mass index $\left(\mathrm{kg} / \mathrm{m}^{2}\right)$ & $25.1(22.5-28.1)$ & $25.1(21.9-28.5)$ & $25.0(23.2-27.7)$ & $0.495^{b}$ \\
\hline Obesity & $11(18.6)$ & $5(16.7)$ & $6(20.7)$ & 0.692 \\
\hline Hypertension & $23(35.9)$ & $12(38.7)$ & $11(33.3)$ & 0.654 \\
\hline Diabetes & $7(10.9)$ & $3(9.7)$ & $4(12.1)$ & 1.000 \\
\hline Smoker & $5(7.8)$ & $3(9.7)$ & $2(6.1)$ & 0.667 \\
\hline ASA & $2(2-3)$ & $2(2-3)$ & $2(2-3)$ & 0.486 \\
\hline$A S A \geq 3$ & $22(33.8)$ & $13(40.6)$ & $9(27.3)$ & 0.255 \\
\hline \multicolumn{5}{|l|}{ Preoperative clinical status } \\
\hline Alteration of the $L O C$ & $10(15.2)$ & $7(21.9)$ & $3(8.8)$ & 0.180 \\
\hline Motor deficit & & & & 0.696 \\
\hline Not present & $35(53.0)$ & $17(53.1)$ & $18(52.9)$ & \\
\hline Light (grade IV MRC) & $16(24.2)$ & $9(28.1)$ & $7(20.6)$ & \\
\hline Severe (grade III MRC or less) & $15(22.7)$ & $6(18.8)$ & $9(26.5)$ & \\
\hline Dysphagia & $1(1.5)$ & $0(0.0)$ & $1(3.0)$ & 1.000 \\
\hline KPS $<70 \%$ & $20(30.3)$ & $11(34.4)$ & $9(26.5)$ & 0.485 \\
\hline$E C O G \geq 3$ & $14(21.2)$ & $9(28.1)$ & $5(14.7)$ & 0.183 \\
\hline
\end{tabular}

Data presented as $\mathrm{n}(\%)$, except for age (mean \pm standard deviation) and body mass index (median and quartiles)

${ }^{\text {a }}$ Student T test; ${ }^{\mathrm{b}}$ Mann-Whitney test

ECOG Eastern Cooperative Oncology Group, KPS Karnofsky Performance Scale, LOC level of consciousness, MRC Medical Research Council Scale

tract infection and one had an isolated seizure on immediate post-operative period without any other neurological alterations). All the three cases of reoperations occurred in DAHD group, although this difference in incidence did not reach statistical significance $(p=0.239)$. None of the reoperations were due to late complications or beyond the 2nd POD. Severe sepsis was the most common cause of major complication in both groups, whereas seizures represented the main cause among the minor complication ones. Thirty-day return to the emergency department was very common in both groups: Pre-DAHD group - 7 (21.9\%) patients and DAHD group - $10(29.4 \%)$ patients, $p=0.484$, which lead to six (18.8\%) readmissions in Pre-DAHD and three $(9.4 \%)$ in DAHD group, $p=0.474$. Thirty-day mortality rate was $4.6 \%$ and did not differ between the groups: Pre-DAHD group -1 (3.1\%) patient vs DAHD group $-2(6.1 \%)$ patients, $p=1.000$.

Table 6 compares the costs of treatment between the two groups. There was a significant reduction in the costs of hospitalization in DAHD group (US\$2135 [quartiles US\$1472 - US\$3800] vs US\$2765 [quartiles US\$2185 -
US\$4333], $p=0.043)$. Figure 3 depicts the distribution of costs in the two groups. While ICU costs were similar between the groups $(p=0.383)$, ward costs were significantly lower in DAHD group (US\$922 [quartiles US\$658 US\$1798] vs US\$1623 [quartiles US\$1155 - US\$2507], $p=0.009$ ). Thirty-day outpatient costs and 30-day overall costs were consonant between the two groups $(p=0.734$ and $p=0.112$, respectively).

\section{Discussion}

In this cohort of brain tumour patients, the adoption of the DAHD following surgery reduced postoperative LOS, with no increases in complication rates compared with traditional management after surgery.

Traditionally, all patients submitted to craniotomy for brain tumour resection in our institution were kept inpatient at least 4 days for neurological surveillance. This behaviour follows a worldwide trend in neurosurgical care, which has averages of intrahospital stays in the postoperative neurosurgical period ranging from 4 to 6 days $[5,12]$. 
Table 2 Characterization of tumor and surgery

\begin{tabular}{|c|c|c|c|c|}
\hline Variables & General (66) & Pre-DAHD (32) & DAHD (34) & $\mathrm{p}$ \\
\hline \multicolumn{5}{|l|}{ Tumor characteristics } \\
\hline Histology & & & & 0.455 \\
\hline Low grade glioma & $11(16.9)$ & $4(12.5)$ & $6(17.6)$ & \\
\hline High-grade glioma & $25(38.5)$ & $14(43.8)$ & $11(32.4)$ & \\
\hline Metastasis & $28(43.1)$ & $13(40.6)$ & $16(47.1)$ & \\
\hline Meningothelial & $1(1.5)$ & $0(0.0)$ & $1(2.9)$ & \\
\hline Inflammatory process & $1(1.5)$ & $1(3.1)$ & $0(0.0)$ & \\
\hline Previous chemotherapy & $22(33.8)$ & $9(29.0)$ & $13(38.2)$ & 0.434 \\
\hline Previous radiotherapy & $12(18.2)$ & $7(21.9)$ & $5(14.7)$ & 0.450 \\
\hline \multicolumn{5}{|l|}{ Number lesions resected } \\
\hline 1 & $61(92.4)$ & $31(96.9)$ & $30(88.2)$ & 0.387 \\
\hline 2 & $3(4.5)$ & $0(0.0)$ & $3(8.8)$ & \\
\hline 3 or more & $2(3.0)$ & $1(3.1)$ & $1(2.9)$ & \\
\hline Larger lesion diameter (mm) & $44.5(30.8-54.3)$ & $47.5(32.3-58.0)$ & $41.0(30.0-52.5)$ & $0.345^{a}$ \\
\hline Infratentorial location & $5(7.6)$ & $2(6.3)$ & $3(8.8)$ & 1.000 \\
\hline Depth $>1 \mathrm{~cm}$ of the cortex & $33(50.8)$ & $16(51.6)$ & $17(50.0)$ & 0.897 \\
\hline Primary motor area involvement & $15(22.7)$ & $7(21.9)$ & $8(23.5)$ & 0.873 \\
\hline Laterality & & & & 0.707 \\
\hline Right & $25(37.9)$ & $10(31.3)$ & $15(44.1)$ & \\
\hline Left & $34(51.5)$ & $18(56.3)$ & $16(47.1)$ & \\
\hline Bilateral & $3(4.5)$ & $2(6.3)$ & $1(2.9)$ & \\
\hline Posterior Fossa & $4(6.1)$ & $2(6.3)$ & $2(5.9)$ & \\
\hline Edema & & & & 0.064 \\
\hline Absent & $15(22.7)$ & $4(12.5)$ & $11(32.4)$ & \\
\hline Little edema & $29(43.9)$ & $15(46.9)$ & $14(41.2)$ & \\
\hline Very edema & $22(33.3)$ & $13(40.6)$ & $9(26.5)$ & \\
\hline Herniation & $35(53.0)$ & $15(46.9)$ & $20(58.8)$ & 0.331 \\
\hline \multicolumn{5}{|l|}{ Characterization of surgery } \\
\hline Emergency surgery & $5(7.6)$ & $2(6.3)$ & $3(8.8)$ & 1.000 \\
\hline Awake Surgery & $3(4.6)$ & $1(3.1)$ & $2(6.1)$ & 1.000 \\
\hline Duration of surgery (min) & $213(165-291)$ & $213(155-298)$ & 218 (169-283) & $0.773^{a}$ \\
\hline Duration of anesthesia (min) & $325(285-414)$ & $315(281-429)$ & 335 (289-403) & $0.667^{a}$ \\
\hline
\end{tabular}

Data presented as $\mathrm{n}(\%)$, except for lesion diameter (medians and quartiles)

${ }^{a}$ Mann-Whitney test

This study shed light on the shift in perioperative care with the adoption of quality improvement programs such as ERAS and Fast-track surgery and the need to evaluate the safety of these approaches in the surgical fields. We have adopted the DAHD in our Neurooncology department based on the experience of other brain tumour centre, [4] developed from the observation of an uncontrolled case series. Differently from the study that originated the DAHD, [4] we performed a historical cohort comparing two similar groups of patients, candidates for brain tumour resection before and after the implementation of DAHD. The high comparability between the groups gives greater reliability to our results and raises validity to the method.

Although there was a reduction of hospital LOS (median) from 5 days to 3 days after implantation of the protocol, the LOS in the ICU did not change. We believe that this is justified because all patients were assisted in the ICU bed after surgery and the assisting surgeons were not responsible for the clinical care adopted during this stay, so the algorithm could only be applied after the patient has been discharged from the ICU. Therefore, we believe that the hospital LOS could be even shorter if patients were assisted in a postoperative ward 
Table 3 Characterization of outcomes

\begin{tabular}{llll}
\hline Variables & General (66) & Pre (32) & Post (34) \\
\hline Extent of resection & $52(78.8)$ & $23(71.9)$ & $29(85.3)$ \\
GTR & $13(19.7)$ & $9(28.1)$ & $4(11.8)$ \\
STR & $1(1.5)$ & $0(0.0)$ & $1(2.9)$ \\
Partial / Biopsy & & & $24(70.6)$ \\
Postoperative motor status & $44(67.7)$ & $20(64.5)$ & $5(14.7)$ \\
$\quad$ Maintained & $11(16.9)$ & $6(19.4)$ & $1(2.9)$ \\
Improvement & $4(6.2)$ & $3(9.7)$ & $4(11.8)$ \\
New mild deficit & $6(9.2)$ & $2(6.5)$ & $3(8.8)$ \\
New severe deficit & $6(9.2)$ & $3(9.7)$ & \\
Post-operative dysphagia & & $1(3.1)$ & $16(47.1)$ \\
LOS & $17(25.8)$ & $5(4-8)$ & $3(2-5)$ \\
Early discharge (\%) & $4(2-7)$ & $28.8(22.7-67.1)$ & $26.5(23.5-50.9)$ \\
LOS (days) & $26.6(23.3-52.0)$ & $0.25(0.15-0.46)$ & $0.44(0.33-0.65)$ \\
ICU stay (hours) & $0.38(0.18-0.51)$ & & 0.928 \\
Proportion of ICU stay / LOS &
\end{tabular}

Categorical variables are presented as $\mathrm{n}(\%)$ and continuous as medians and quartiles

${ }^{a}$ Mann-Whitney test

GTR gross total resection, ICU intensive care unit, LOS Length of Stay, STR subtotal resection

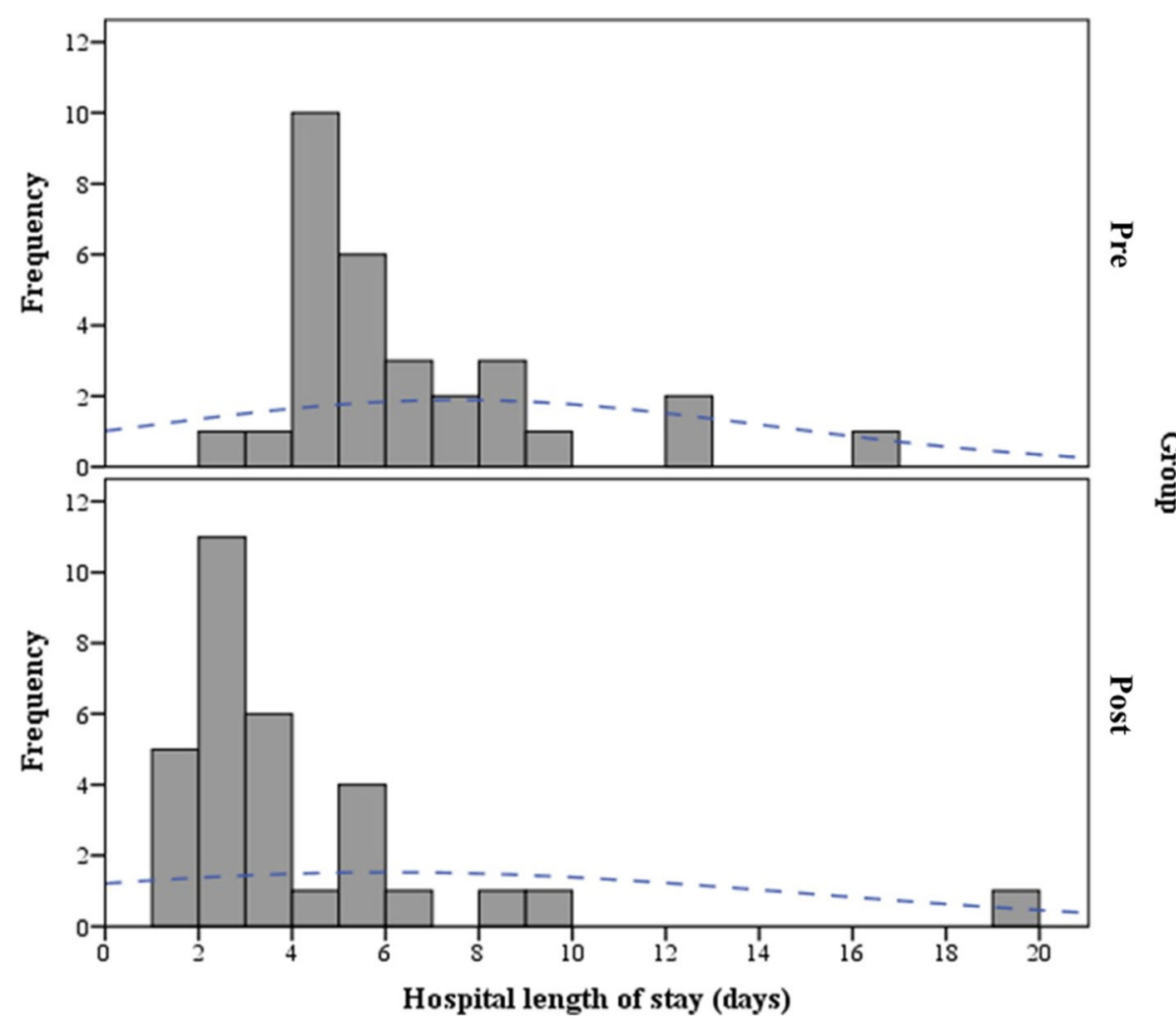

Fig. 2 Histogram of length of hospitalization before (Pre-DAHD) and after DAHD implementation is shown above and below, respectively. There is an increase in the frequency of early $(<48 \mathrm{~h})$ discharge after DAHD adoption 
Table 4 Multivariate analysis by logistic regression to fit the patient's performance

\begin{tabular}{llllll}
\hline Variables & Coefficient \pm SE & Wald & OR & Cl 95\% & $\boldsymbol{p}$ value \\
\hline DAHD (vs Pre-DAHD) & $3.36 \pm 1.09$ & 9.61 & 28.92 & $3.45-242.78$ & 0.002 \\
KPS $<70$ (vs $\geq 70)$ & $-1.58 \pm 0.88$ & 3.22 & 0.21 & $0.04-1.16$ & 0.073
\end{tabular}

CI Confidence interval, DAHD Daily algorithm for hospital discharge, KPS Karnofsky Performance Scale, OR Odds ratio, SE Standard error

bed, which has been shown to be safe in other studies $[6,9]$. In fact, routine outpatient care following brain tumour resection has been proved to be safe in selected patients $[6,10,13]$, although patients with greater risks (large tumours, posterior fossa, worse status performance) were excluded. Sughrue et al., who included all patients with brain tumours at their institution, assisted patients in an intermediate care unit [4]. The use of protocols to identify patients at greater risk for complications or need for prolonged ICU stay (e.g. advanced age, diabetes, high intraoperative blood losses, and longer surgical procedures) could reduce the number of unnecessarily assisted ICU patients and may shorten the LOS $[14,15]$.

Table 5 Complication and safety outcomes

\begin{tabular}{|c|c|c|c|c|}
\hline Outcomes $^{\mathbf{a}}$ & General (66) & Pre (32) & Post (34) & $p$ \\
\hline Reoperations & $3^{\mathrm{a}}(4.5)$ & $0(0.0)$ & $3(8.8)$ & 0.239 \\
\hline Hematoma & $2(3.0)$ & $0(0.0)$ & $2(5.9)$ & 0.493 \\
\hline Acute hydrocephalus & $1(1.5)$ & $0(0.0)$ & $1(2.9)$ & 1.000 \\
\hline Brain edema & $1(1.5)$ & $0(0.0)$ & $1(2.9)$ & 1.000 \\
\hline Major complication & $10(15.2)$ & $6(18.8)$ & $4(11.8)$ & 0.505 \\
\hline Stroke & $2(3.0)$ & $1(3.1)$ & $1(2.9)$ & 1.000 \\
\hline ACS & $(0.0)$ & $(0.0)$ & $(0.0)$ & - \\
\hline PT & $1(1.5)$ & $1(3.1)$ & $0(0.0)$ & 0.485 \\
\hline Severe sepsis & $8(12.1)$ & $5(15.6)$ & $3(8.8)$ & 0.469 \\
\hline Meningitis & $2(3.0)$ & $2(6.3)$ & $0(0.0)$ & 0.231 \\
\hline State of epilepsy & $2(3.0)$ & $2(6.3)$ & $0(0.0)$ & 0.231 \\
\hline Minor complication & $11(16.7)$ & $6(18.8)$ & $5(14.7)$ & 0.660 \\
\hline Urinary tract infection & $2(3.0)$ & $1(3.1)$ & $1(2.9)$ & 1.000 \\
\hline Pneumonia & $(0.0)$ & $(0.0)$ & $(0.0)$ & - \\
\hline Deep vein thrombosis & $3(4.5)$ & $2(6.3)$ & $1(2.9)$ & 0.608 \\
\hline Surgical wound infection & $1(1.5)$ & $1(3.1)$ & $0(0.0)$ & 0.485 \\
\hline Dehiscence & $2(3.0)$ & $2(6.3)$ & $0(0.0)$ & 0.231 \\
\hline CSF Fistula & $4(6.1)$ & $4(12.5)$ & $0(0.0)$ & 0.05 \\
\hline Seizures & $7(10.6)$ & $4(12.5)$ & $3(8.8)$ & 0.705 \\
\hline Return to the ED & $17(25.8)$ & $7(21.9)$ & $10(29.4)$ & 0.484 \\
\hline Readmissions & $9(14.1)$ & $6(18.8)$ & $3(9.4)$ & 0.474 \\
\hline Death & $3(4.6)$ & $1(3.1)$ & $2(6.1)$ & 1.000 \\
\hline
\end{tabular}

The data is displayed as $n(\%)$

${ }^{a}$ One patient was reoperated because of hematoma and cerebral edema Legend: ${ }^{\text {a }}$ Outcomes occurred until the 30th postoperative day. ACS acute coronary syndrome, CSF cerebrospinal fluid, ED emergency department, $P T$ pulmonary thromboembolism
Healthcare costs were lower in the DAHD group during hospitalization mainly due to the reduction of nursing costs. Thirty-day costs, despite a tendency towards lower costs in the DAHD group, were not significant, although the current study may be underpowered for this outcome. Other studies that evaluated Fast-track protocols, due to a lack of control group, did not perform such comparisons $[4,6-9,13]$.

Neurosurgical complications are quite common after brain tumour surgeries and these are the main reason for longer periods of LOS. The most common complications reported in the literature are venous thromboembolism, new or worsened neurological deficit, dural closure-related complications, postoperative peritumoural brain oedema, early postoperative seizure, general medical complications, wound infection, and surgery-related hematoma $[5,16,17]$. In our study, we had a higher incidence of complications (15.2\% of major complications and $16.7 \%$ of minor complications) when compared to other similar case series, $[4,6-9,11,13]$ which may be due to many different factors: 1 - most of the studies selected patients with safer profiles for outpatient or early discharge surgery, and excluded patients with worsen performance status, large tumours or posterior fossa lesions; 2 - this study was carried out in a tertiary referral hospital of a developing country, where patients submitted to neurosurgical treatment may have already severe neurological deficits, poor performance status, and greater volume of systemic disease (in cases of brain metastasis); 3 - our sample is composed mostly of malignant tumours (metastasis and high grade glioma comprised $81.6 \%$ of the sample), theoretically more prone to postoperative complications [18].

Reduction of costs has been pursued for many healthcare systems worldwide. Within this framework, shorter LOS and reduction in readmissions have been relevant outcomes. Thirty-day readmission rates have been used as a quality of healthcare indicator, with readmissions being associated with increased hospital costs and mortality [18-21]. We reported a high hospital readmission rate (14.1\%), which may be explained by the large number of patients with poor performance status before neurological surgery ( $30 \%$ of patients with KPS $<70$ ). However, complications, readmissions, and mortality rate were similar between the two groups, which supports that early discharge was safe and feasible even in our population profile.

There are several limitations of this study. This was a single-centre study with a small sample size, although 
Table 6 Costs of treatment between the two groups

\begin{tabular}{|c|c|c|c|c|}
\hline Variables & General (66) & Pre (32) & Post (34) & $p$ \\
\hline Costs of hospitalization & $2567(1642-4137)$ & $2765(2185-4333)$ & $2135(1472-3800)$ & 0.043 \\
\hline Ward & $1436(763-2311)$ & 1623 (1155-2507) & $922(657-1798)$ & 0.009 \\
\hline $\mathrm{ICU}$ & $817(741-1661)$ & $1472(747-2100)$ & $807(741-1614)$ & 0.383 \\
\hline \multicolumn{5}{|l|}{ 30-day outpatient costs } \\
\hline Clinic & $43(35-87)$ & $45(0-85)$ & $43(40-87)$ & 0.734 \\
\hline Emergency department & $0(0-537)$ & $0(0-0)$ & $0(0-613)$ & 0.356 \\
\hline Overall costs until 30th POD & $2963(2041-4624)$ & 3563 (2290-4915) & $2511(1796-4132)$ & 0.112 \\
\hline
\end{tabular}

Legend: Data are presented as median and interquartile interval in dollars (US\$) Intrahospitalar deaths were excluded from the analysis of outpatient costs The Mann-Whitney test was used for all comparisons $I C U$ intensive care unit, $P O D$ postoperative day;

our population was homogeneous. The study design, with cases and controls patients selected in different time frames, may contribute to biases, such as subtle increases of surgeon's and multidisciplinary team experiences or other structural changes in the service that may have occurred during the different periods. Yet, the time frame of our study was short, which may have minimized such limitation. Moreover, seasonality issues and wash-out period, commonly applied and evaluated in before-after studies, were not investigated. Nevertheless, DAHD feasibility and safety were the main goals of this study. We believe that most patients and their relatives were fulfilled with early discharge, although it was not possible to retrospectively measure the level of satisfaction, which may be objective of future studies.

In 2018, Wang et al published the first prospective clinical trial evaluating the application of an ERAS protocol in cranial surgeries, showing significant benefits over conventional perioperative care, including reduction in postoperative LOS and faster recovery [11]. However, the adoption of this protocol required a large number of multidisciplinary professionals, including the need of a paradigm shift in the whole team, which makes their prompt reproducibility difficult to achieve in other settings. Our work provides feasible, not expensive small changes that can be adopted in any neurosurgical service

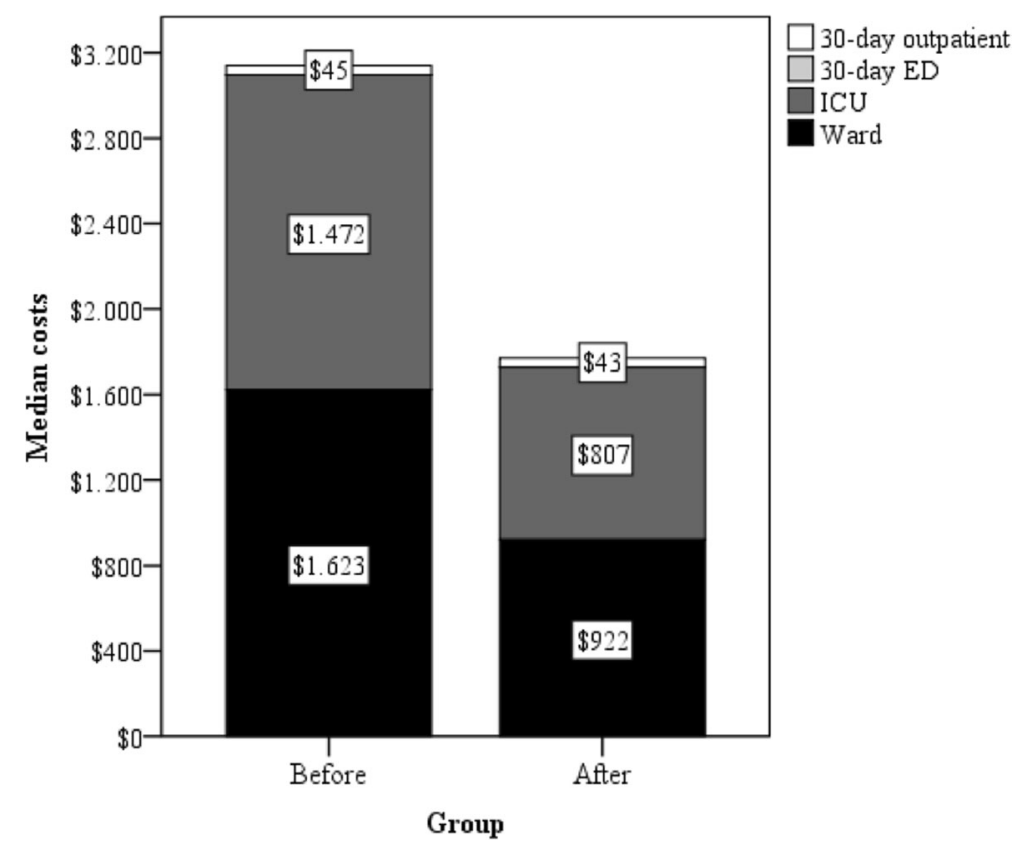

Fig. 3 Distribution of 30-day inpatient and outpatient healthcare costs following brain tumor. This figure compares the distribution of 30-day healthcare costs between the two groups. Regarding ward costs, significant lower expenses were seen after DAH 
before a greater multidisciplinary ERAS protocol could be adopted.

\section{Conclusion}

The implementation of a standardized protocol for hospital discharge of patients submitted to brain tumour surgery led to a significant reduction in hospital LOS, reducing hospitalization costs, not increasing readmission rate or postoperative complications.

\section{Abbreviations}

DAHD: Daily Algorithm For Hospital Discharge; ECOG: Eastern Cooperative Oncology Group; ERAS: Enhanced Recovery After Surgery; ICU: Intensive Care Unit; KPS: Karnofsky Performance Status; LOS: Lenght Of Stay; SPSS: Statistical Package For Social Sciences

\section{Acknowledgements}

Not applicable.

\section{Authors' contributions}

Concept and design: ISN. Acquisition, analysis or interpretation of data: ISN, FMU, DGQ, DJFS. Drafting of the manuscript: ISN, FMU, DGQ, DJFS, MFL. Critical revision of the manuscript for important intellectual content: ISN, MFL, CMS, EV, URJ, RLOA, WSP, MJT. Statistical analysis: DJFS. Supervision: WSP, MJT. All authors had full access to all data in the study and take responsibility for the integrity of the data and the accuracy of the data analysis. All authors have read and approved the manuscript.

\section{Funding}

No funding was received for this research.

\section{Availability of data and materials}

The datasets used and/or analysed during the current study are available from the corresponding author on reasonable request.

\section{Ethics approval and consent to participate}

All procedures performed in studies involving human participants were in accordance with the ethical standards of the institutional research committee (USP - Faculdade de Medicina da Universidade de São Paulo FMUSP - Instituto do Câncer do Estado de São Paulo Ethics and Research Committee - CAAE 87064218.2.0000.0065) and with the 1964 Helsinki declaration and its later amendments or comparable ethical standards.

\section{Consent for publication}

Not applicable.

\section{Competing interests}

All authors certify that they have no affiliations with or involvement in any organization or entity with any financial interest (such as honoraria; educational grants; participation in speakers' bureaus; membership, employment, consultancies, stock ownership, or other equity interest; and expert testimony or patent-licensing arrangements), or non-financial interest (such as personal or professional relationships, affiliations, knowledge or beliefs) in the subject matter or materials discussed in this manuscript.

\section{Author details}

'Instituto do Cancer do Estado de São Paulo do Hospital das Clinicas da Faculdade de Medicina da Universidade de São Paulo, Av Dr Arnaldo 251 Cerqueira Cesar, CEP, São Paulo 01246-000, Brazil. 'Division of Neurosurgery, Hospital das Clinicas da Faculdade de Medicina da Universidade de São Paulo, Av Dr Arnaldo 251 Cerqueira Cesar, CEP, São Paulo 01246-000, Brazil. ${ }^{3}$ Division of Anaesthesiology, Hospital São Paulo, Universidade Federal de São Paulo, São Paulo, Brazil. ${ }^{4}$ Financial, Planning, and Control Board, Instituto do Cancer do Estado de São Paulo do Hospital das Clinicas da Faculdade de Medicina da Universidade de São Paulo, São Paulo, Brazil. ${ }^{5}$ Department of Gastroenterology, Faculdade de Medicina da Universidade de São Paulo, São Paulo, Brazil. ${ }^{6}$ Universidade Federal do Amazonas, Manaus, Brazil.
Received: 10 February 2020 Accepted: 5 May 2020

Published online: 14 May 2020

\section{References}

1. Engelman RM, Rousou JA, Flack JE, Deaton DW, Humphrey CB, Ellison LH, Allmendinger PD, Owen SG, Pekow PS. Fast-track recovery of the coronary bypass patient. Ann Thorac Surg. 1994;58(6):1742-6.

2. Ljungqvist $\mathrm{O}$, Scott M, Fearon KC. Enhanced recovery after surgery: a review. JAMA Surg. 2017;152(3):292.

3. Fearon $\mathrm{KCH}$, Ljungqvist $\mathrm{O}$, Von Meyenfeldt $\mathrm{M}$, et al. Enhanced recovery after surgery: a consensus review of clinical care for patients undergoing colonic resection. Clin Nutr. 2005;24(3):466-77.

4. Sughrue ME, Bonney PA, Choi L, Teo C. Early discharge after surgery for intra-axial brain Tumours. World Neurosurg. 2015;84(2):505-10.

5. Dasenbrock HH, Liu KX, Devine CA, Chavakula V, Smith TR, Gormley WB, Dunn IF. Length of hospital stay after craniotomy for tumour: a National Surgical Quality Improvement Program analysis. Neurosurg Focus. 2015; 39(6):E12.

6. Au K, Bharadwaj S, Venkatraghavan L, Bernstein M. Outpatient brain tumour craniotomy under general anesthesia. J Neurosurg. 2016;125(5):1130-5.

7. Boulton M, Bernstein M. Outpatient brain tumour surgery: innovation in surgical neurooncology. J Neurosurg. 2008;108(4):649-54.

8. Purzner T, Purzner J, Massicotte EM, Bernstein M. Outpatient brain tumour surgery and spinal decompression: a prospective study of 1003 patients. Neurosurgery. 2011;69(1):119-27.

9. Ma R, Livermore LJ, Plaha P. Fast track recovery program after endoscopic and awake Intraparenchymal brain tumour surgery. World Neurosurg. 2016; 93:246-52.

10. Venkatraghavan L, Bharadwaj S, Au K, Bernstein M, Manninen P. Same-day discharge after craniotomy for supratentorial tumour surgery: a retrospective observational single-Centre study. Can J Anesth Can Anesth. 2016;63(11):1245-57.

11. Wang Y, Liu B, Zhao T, et al. Safety and efficacy of a novel neurosurgical enhanced recovery after surgery protocol for elective craniotomy: a prospective randomized controlled trial. J Neurosurg. 2019;130(5):1680-91.

12. Marcus LP, McCutcheon BA, Noorbakhsh A, Parina RP, Gonda DD, Chen C, Chang DC, Carter BS. Incidence and predictors of 30-day readmission for patients discharged home after craniotomy for malignant supratentorial tumours in California (1995-2010). J Neurosurg. 2014;120(5):1201-11.

13. Grundy PL, Weidmann C, Bernstein M. Day-case neurosurgery for brain tumours: the early United Kingdom experience. Br J Neurosurg. 2008;22(3): 360-7.

14. Hanak BW, Walcott BP, Nahed BV, Muzikansky A, Mian MK, Kimberly WT, Curry WT. Postoperative intensive care unit requirements after elective craniotomy. World Neurosurg. 2014;81(1):165-72.

15. Ziai WC, Varelas PN, Zeger SL, Mirski MA, Ulatowski JA. Neurologic intensive care resource use after brain tumour surgery: an analysis of indications and alternative strategies. Crit Care Med. 2003;31(12):2782-7.

16. Sawaya R, Hammoud M, Schoppa D, Hess KR, Wu SZ, Shi WM, Wildrick DM. Neurosurgical outcomes in a modern series of 400 craniotomies for treatment of parenchymal tumours. Neurosurgery. 1998;42(5):1044-55 discussion 1055-1056.

17. Wong JM, Panchmatia JR, Ziewacz JE, Bader AM, Dunn IF, Laws ER, Gawande AA. Patterns in neurosurgical adverse events: intracranial neoplasm surgery. Neurosurg Focus. 2012;33(5):E16.

18. Nuño M, Ly D, Ortega A, Sarmiento JM, Mukherjee D, Black KL, Patil CG. Does 30-Day Readmission Affect Long-term Outcome Among Glioblastoma Patients? Neurosurgery. 2014;74(2):196-205.

19. Dickinson H, Carico C, Nuño M, Mukherjee D, Ortega A, Black KL, Patil CG. Unplanned readmissions and survival following brain tumour surgery. J Neurosurg. 2015;122(1):61-8.

20. Elsamadicy AA, Sergesketter A, Adogwa O, Ongele M, Gottfried ON. Complications and 30-day readmission rates after craniotomy/craniectomy: a single institutional study of 243 consecutive patients. J Clin Neurosci. 2018;47:178-82.

21. Tsai TC, Joynt KE, Orav EJ, Gawande AA, Jha AK. Variation in surgicalreadmission rates and quality of hospital care. N Engl J Med. 2013;369(12): $1134-42$.

\section{Publisher's Note}

Springer Nature remains neutral with regard to jurisdictional claims in published maps and institutional affiliations. 